\title{
Research on AMR Sensors' Anti-interference Circuit
}

\author{
Qi Zhang a, Yingtang Zhang ${ }^{\mathrm{b}}$ and Zhining $\mathrm{Li}^{\mathrm{c}}$ \\ Department of Vehicles and Electrical Engineer, Ordnance Engineering College, Shijiazhuang \\ 050003, China \\ aqiqi.12321@qq.com, bzyt01@mails.tsinghua.edu.cn, clizn03@mails.tsinghua.edu.cn
}

Keywords: Anisotropic Magneto resistive Sensor; HMC2003; Set/Reset Circuit; Anti-interference.

\begin{abstract}
Aiming at the problems of the decline of both accuracy and sensitivity of the anisotropic magneto resistive (AMR) sensors caused by outside interference, this paper puts forward the applied set or reset pulse to generate strong magnetic field to restore and solve the problem, and takes 3-axis AMR sensor HMC2003 as an example, introducing its basic parameters, analyzing the working principle of Set/Reset (SR) pulse and simulating the SR circuit composed of filed effect transistors. Finally, relevant experiments are done with this circuit. The experimental results show that the sensor with SR circuit has a stronger anti-interference capability and a higher detection accuracy than the one without SR circuit in the geomagnetic field.
\end{abstract}

\section{Introduction}

Anisotropic magneto resistive (AMR) sensors have advantages of wide measuring range, high sensitivity, small size and low cost [1], and have been applied in the field of weak-magnetic detection. To determine the characteristics of plasma coagulation, Cuicui Min [2] utilized single-axis AMR sensor HMC1021Z to detect the movement and time of the bead track. For the detection of magnetic capsule in vivo movement, Zhaorui Zeng [3] constituted a three-axis AMR sensor array with a single-axis AMR sensor HMC1021 and a dual-axis AMR sensor HMC1022. Zhongchao Qiu [4] utilized the above-mentioned three-axis AMR sensor array and obtained the shape and position information of prefabricated crack on the plate. The above methods provide a reference for building a detection system based on AMR sensor array. However, affected by external interference, the maximum resolution of AMR sensor is less than $2 \mathrm{nT}$, which would result in inaccurate detection results. This paper describes the working process of the SR circuit with the three-axis AMR sensor HMC2003, and validates the effectiveness of the SR circuit in improving the anti-jamming ability.

\section{Theory}

\subsection{HMC 2003}

Based on the characteristics of the resistivity of the ferromagnetic material varying with the angle between the internal magnetization direction and the current direction, the basic unit of AMR sensor is a long and thin perm-alloy. When the external magnetic field changes, the resistance of the alloy band changes by the form of output voltages [5]. This paper uses a three-axis AMR sensor HMC2003, whose magnetic field detection range is \pm 2 Gauss. The resolution is $40 \mathrm{nT}$ and the sensitivity is $1 \mathrm{~V} / \mathrm{Gauss}$. The output range of the three axis ( $\mathrm{X}, \mathrm{Y}$ and $\mathrm{Z}$ axis) is from $0.5 \mathrm{~V}$ to $4.5 \mathrm{~V}$. When the magnetic induction intensity is zero, the three-axis typical output and reference voltage values are $2.5 \mathrm{~V}$ [6].

\subsection{SR circuit}

AMR sensors are susceptible to external interference, leading to reduced accuracy and sensitivity, and zero drift [7]. In order to improve the anti-jamming capability of AMR sensors, SR pulses are applied to the AMR sensors by SR currents. Then a strong magnetic field is generated to reunify the magnetic domain inside the sensor in one direction to ensure that the sensor is always in the most sensitive state.

Affected by the external magnetic material, the internal magnetic domain of AMR sensor is disturbed. When a set pulse is successfully applied, the internal magnetic domain will be unified to the positive 
direction of the magnetization axis, then the bias voltage of the bridge corresponds to a positive slope of the output. In contrast, when a reset pulse is successfully applied to the sensor, its internal magnetic domain will converge to the reverse of the magnetization axis, then the bias voltage of the bridge will have a negative slope for the output. The set pulse and reset pulse have the same principle, while the only difference is the output direction [8], the expression is written as follows

$$
\begin{aligned}
& V_{\text {set }}=S \cdot H+V_{\text {off }} \\
& V_{\text {reset }}=-S \cdot H+V_{\text {off }}
\end{aligned}
$$

Where, $V_{\text {set }}$ and $V_{\text {reset }}$ represent the output voltage after the set and reset pulse, respectively. $S$ is the sensor sensitivity and $H$ represents the magnetic field strength. $V_{\text {off }}$ is the output drift of the AMR sensor.

\section{Simulations}

The SR current band of the AMR sensor HMC2003 can be considered as a resistor, which is typically $4.5 \Omega$. For the setting or resetting, a current, whose value is 3 to $4 \mathrm{~A}$ and pulse width is at least $2 \mu \mathbf{s}$, is applied to the bismuth alloy film. The voltage required to drive the load of $5 \Omega$ is 15 to $20 \mathrm{~V}$.

The SR pulses are generated by two CMOS tubes, as shown in Fig. 1. The signal generator XFG1 and XFG2 provide two periodic square wave signals with the amplitude of $5 \mathrm{~V}$, the frequency of 20 $\mathrm{KHz}$ and the phase difference of $180^{\circ}$. The set square wave signal is connected to the gate of the $\mathrm{P}$ channel CMOS tube (X2) through the NPN transistor 2N3904 (Q1), while the reset square wave signal is connected to the gate of the $\mathrm{N}$ channel CMOS tube (X1).

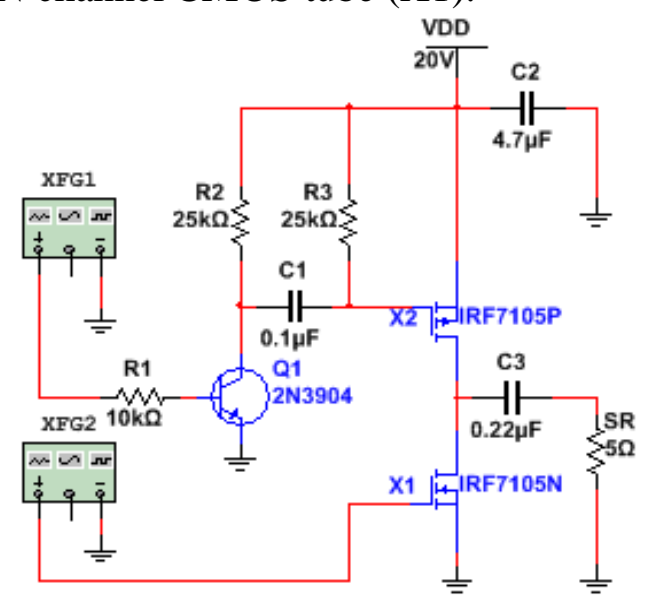

Fig. 1 The diagram of the SR circuit

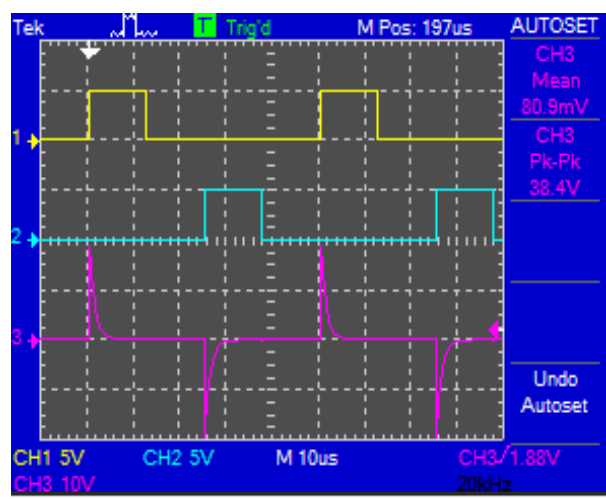

Fig. 2 The waveforms of the SR circuit

Simulation waveform is shown in Fig.2. No. 1 and No. 2 waveform represent the input set square wave signal and reset square wave signal, respectively. No. 3 waveform shows the output of the S R pulse signal. When the set signal is in low level, Q1 is off. Both sides of the capacitor C1 are in high level. $\mathrm{X} 2$ gate is in high level and there is no voltage between the source and the gate, so there is no current 
output. When the reset signal is in low level, there is no voltage between the source and the gate of X1, so there is no current output. When the set signal changes from low level to high level, Q1 turns on and the left side of $\mathrm{C} 1$ turns low. Because $\mathrm{C} 1$ has not been completed charged, the right side of $\mathrm{C} 1$ is in low level. Then X2 is on while X1 is off. VDD charges C3 through X2 to generate a set pulse. The capacitor $\mathrm{C} 3$ discharges through $\mathrm{X} 1$, resulting in a reset pulse.

\section{Experiment}

The experimental device consists of a signal generator, an oscilloscope, a data acquisition instrument, conditioning circuits, PC and other components. The influence of SR circuit on the accuracy of AMR sensor is analyzed by experiments in geomagnetic field.

The experiment steps are as follows. First, fix the sensor HMC2003 and supply power to the sensor to make it work normally. Measure the reference voltage with the data acquisition instrument, and then collect the output signal of $\mathrm{X}, \mathrm{Y}$ and $\mathrm{Z}$ axis respectively. The average output voltage of the three axes is obtained. Collect the data every five seconds, and repeat the collection for 20 times. Second, repeat the above steps with the SR circuit. Third, organize, process and plot the obtained data. The experimental results are shown in Fig. 3.

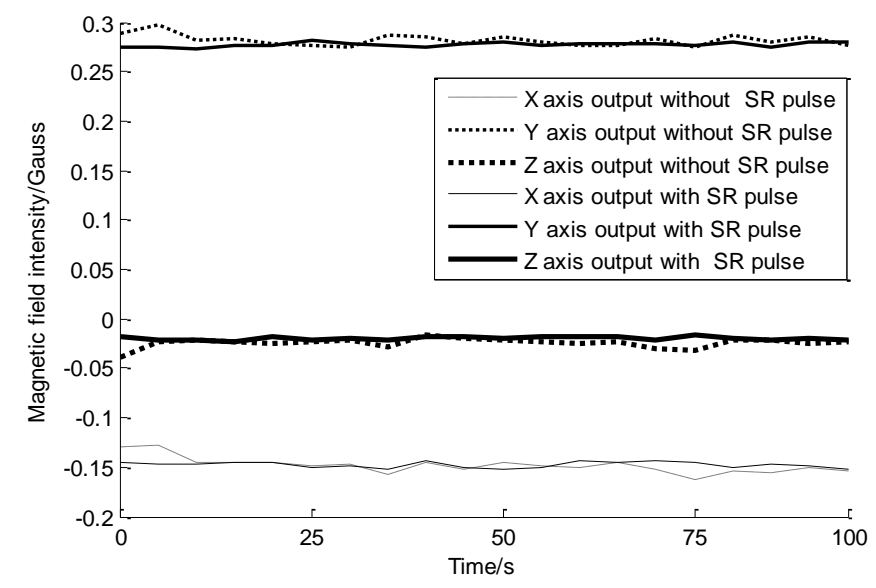

Fig.3. The measured magnetic field strength with and without the SR circuit

Based on the three-axis direction of the sensor, the geomagnetic field data are as follows: the magnetic field strength in the $\mathrm{X}$-axis positive direction, the $\mathrm{Y}$-axis positive direction and the $\mathrm{Z}$-axis positive direction are -0.148 Gs, 0.285 Gs and -0.020 Gs, respectively.

As can be seen from Fig.3, both circuits can work under the geomagnetic environment. However, the measured magnetic field strength with the SR circuit has a smaller variance than the one without the SR circuit. Additionally, the three output curves of the former are more stable and acurrate. It is shown that adding the SR circuit to the AMR sensor can improve its detection precision under the same magnetic field environment.

\section{Summary}

In this paper, the working principle of AMR sensor HMC2003 and SR circuit are introduced, then the related experiment is carried out. The experimental results show that the SR circuit can help eliminate the external disturbance and improve the detection precision under the environment of geomagnetic field. To further verify the proposed method, we need to study the calibration method of AMR sensor and obtain the parameters such as its accuracy and sensitivity.

\section{References}

[1] Pei Yi, Yu Nanfang, Liu Qi, Theory and application of anisotropic magnetoresistive sensor[J], Instrument Technique and Sensor, 2004 (8): 26-27.

[2] Min Cuicui, The study of blood coagulation detection technique[D], Chongqing Institute of Technology, 2008. 
[3] Zeng Zhaorui, Liu Xiuquan and Zhang Wei, Research and design of a magnetic field location detection system of capsule endoscope inside the human body[J], Microcomputer Information, 2008, 24(20): 238-240.

[4] Qiu Zhongchao, Zhang Weimin and Yang Xiujiang, 3D weak magnetic signals detecting system based on module combination array[J], Instrument Technique and Sensor, 2015 (7): 10-13.

[5] Tianli, Magnetic field measurement system design for three-dimensional based on permalloy magnetoresistive sensors[D], Dalian Jiaotong University, 2010.

[6] Honeywell HMC2003 Data Book, 2005.

[7] ZHANG Xiaoming, WANG Guohuan and LI Zhengxi, High-precision measuring technique of AMR magnetic sensor[J], Journal of Test And Measurement Technology , 2015, 1: 015.

[8] Liang Zhiyong, The development of stray current detector in coal mine based on magneto resistance effects[D], Taiyuan University of Technology, 2014. 